\title{
LE RÔLE DES VILLES DANS LA FORMATION DES SYSTÈMES LOCAUX D'HABITAT
}

Les études géographiques portant sur l'aspect spatial de l'habitat s'intéressent tout particulièrement à la distribution spatiale de l'habitat, la disposition de cités, le réseau d'habitat, la structure spatiale et le système d'habitat. L'ensemble de ces notions et l'ordre dans lequel elles apparaissent ne sont pas dus au hasard mais illustrent les étapes de la colonisation d'un territoire donné et le degré de maturité de l'habitat.

Le processus de colonisation commence par l'installation dans un endroit tout d'abord d'une cité, puis des cités successives. Isolée au début, avec le temps une cité se met en rapport avec d'autres cités ce qui crée entre elles des liens plus ou moins forts dont le résultat est le réseau d'habitat.

Les cités avec tous leurs liens et fonctions constituent la structure spatiale d'habitat régionale ou nationale. Enfin, le système d'habitat se forme lorsque l'habitat atteint un certain degré de maturité c'est-à-dire lorsque les principaux traits structuraux de même que les facteurs dynamisant la structure en question sont consolidés. Étant donné que le processus de développement et de transformation de l'habitat s'opère de façon inégale dans le temps et l'espace, dans chaque territoire l'habitat est différent du point de vue du degré de développement du système d'habitat. Ainsi, il y a les territoires où se sont formés les systèmes d'habitat, et d'autres où l'habitat est créé au niveau soit de la disposition soit d'un réseau de cités.

Les cinq notions que nous venons d'évoquer forment une structure méthodologique qui pourrait être utile à l'étude de l'habitat d'une région ou d'un pays. D'après B.Malisz, la distribution spatiale de l'habitat est définie par les relations entre les lieux d'habitation et les lieux de travail, les lieux de loisirs, d'approvisionnement, d'éducation, d'activités d'associations et organisations, etc.

La disposition des cités doit être comprise comme la localisation topographique et géographique de chaque cité, de groupements de cités ou bien de toutes les cités - urbaines et rurales - d'un pays ou d'une région.

Le réseau d'habitat est le résultat de la disposition de toutes les cités dans un ordre défini (ce qui se réflète dans la densité de cités), divisées en genres et 
classes (villes et villages, compte tenu de leurs grandeurs respectives), avec tout ce qui relie les cités entre elles (voies de communication, etc.).

La disposition et le réseau d'habitat ce sont les catégories dont le contenu ne fait ressortir les moments dynamiques de l'habitat que dans un degré infime. Elles servent principalement à la description de l'état actuel de la colonisation d'un territoire, d'une région, d'un pays, etc.

Quant à la structure spatiale de l'habitat, c'est une notion contenant les informations sur la disposition des cités, leurs grandeurs (population) et fonctions respectives, de même que sur les relations (liens, compte tenu de leur force) entre les cités particulières. En analysant la structure spatiale de l'habitat, nous n'exposons pas non plus les moments dynamiques mais s'il nous arrive de les faire ressortir, c'est principalement pour expliquer l'état de fait c'est-à-dire l'action des forces ayant dominé la formation d'une structure spatiale concrète.

Le système d'habitat, enfin, est une notion qui se rapporte à un ensemble de cités déjà existantes dans un territoire et l'environnement définis, entre lesquelles il y a une division de fonctions et de rôles; il existe également divers liens - de production (économiques et technologiques) et sociaux. Cette notion comprend les moments dynamiques. Le système d'habitat embrasse la disposition de cités, le réseau d'habitat, et la structure spatiale de l'habitat. L'analyse de ce système vise non seulement à décrire l'état de fait mais également à expliquer les mécanismes qui sont à l'origine de la différentiation du réseau, de la structure spatiale et de la distribution spatiale de l'habitat; il s'agit là des mécanismes qui définissent les modes et le degré de satisfaction des besoins de la population, des mécanismes de la croissance des cités particulières et de ceux du développement du réseau d'habitat.

Évidemment, on peut étudier l'habitat sous tous ses aspects et à une échelle différenciée, depuis un État ou un groupe d'États jusqu'aux petites unités administratives. Étant donné une grande complexité de la structure spatiale de l'habitat et du système d'habitat, les stades de leurs développements respectifs dans différentes parties soit d'un continent soit d'un pays à grande superficie, différentes conditions historiques et sociales, enfin différentes forces motrices du développement - on peut se poser des questions sur le caractère adéquat des notions telles que système d'habitat et structure spatiale de l'habitat dans le contexte d'un continent. G.Lappo est d'avis que dans le territoire de chaque pays suffisamment grand, sans parler des continents, on peut distinguer de nombreux systèmes d'habitat liés plus ou moins entre eux, qui à chaque fois forment un élément (sous-système) dans le cadre des systèmes ou complexes spatiaux-productifs. A M.Lappo d'ajouter qu'un certain nombre de cités restent en dehors de ces systèmes.

Quant à la distribution de l'habitat, il faudrait l'analyser à l'échelle locale et régionale, mais dans le contexte du réseau d'habitat et en tant qu'une partie de la structure spatiale de l'habitat régional et national. A l'intérieur du pays, on peut distinguer un certain nombre de distributions 
d'habitat formées souvent au cours d'un long processus historique de colonisation et d'aménagement du territoire.

En analysant les systèmes d'habitat, nous cherchons à faire ressortir les éléments et les interdépendances formant le réseau, la distribution ou la structure d'habitat; nous mettons en même temps un accent sur les mécanismes qui définissent le caractère et la force des liens entre les éléments particuliers constituant le réseau, la distribution ou la structure d'habitat, de même que sur les facteurs qui expliquent le fonctionnement des formes spatiales déterminées de l'habitat. On peut dire, au figuré, qu'en analysant le système d'habitat, on examine non seulement la morphologie mais également la physiologie de l'habitat. Une telle analyse permet non seulement de décrire et d'expliquer mais en même temps de créer les généralisations qui, tout en apportant de nouvelles données, peuvent servir à la formation d'une partie de la réalité environnante.

Les recherches sur l'habitat menées jusqu'à présent ont permis de connaître les formes et la localisation des cités avec un accent particulier mis sur le réseau et les distributions de l'habitat. Par contre, moins nombreux sont les travaux sur la structure spatiale de l'habitat, et il y a relativement peu de ceux qui analysent les systèmes d'habitat. On pourrait expliquer cet état des choses en rappelant que l'approche systémique est relativement récente, et en évoquant les difficultés méthodiques ainsi que l'accès difficile aux informations et aux matériaux nécessaires aux analyses.

Les géographes polonais sont auteurs d'une vingtaine de travaux sur l'habitat en Pologne, où l'approche systémique a été appliquée. Une sui generis récapitulation de considérations concernant ce domaine a été publiée dans Prace Geograficzne IGiZP PAN, tomes 117, 140 et 152. Cette revue de travaux ne permet pas de se faire une idée précise sur le sujet - il en résulte que les recherches portaient, en premier lieu, sur le système national d'habitat, par contre peu nombreuses sont les approches systémiques à l'échelle régionale et locale (à l'échelle d'une commune ou d'un ensemble de communes).

Il est lieu de signaler ici que dans les travaux portant sur le système national on n'analysait pas l'ainsi dit système intégré d'habitat qui s'étend sur toutes les formes d'habitat mais on s'est concentré sur le système urbain. Nombre de travaux ont vu le jour consacrés aux agglomérations urbaines, aux ainsi dits centres nationaux de croissance, aux grandes villes et aux régions urbaines. Il est absolument nécessaire d'entreprendre les recherches également sur les systèmes régionaux d'habitat et surtout sur les systèmes locaux. Outre la volonté de connaître les règles qui régissent la réalité environnante, cette nécessité présente un côté pragmatique issu de l'abandon de la gestion centralisée aussi bien de l'économie que d'autres aspects de la vie de la société.

Le système national d'habitat est constitué de plus de 56 mille cités rurales groupées en 38735 villages, ceux-ci formant plus de 2100 communes et plus de 820 villes (1990). Parmi ces dernières, plus de 200 
villes et env. 300 communes font partie de systèmes complexes d'habitat formant 18 agglomérations urbaines qui, à elles seules, couvrent $20 \%$ du territoire de la Pologne. Aujourd'hui, l'accent particulier devrait être mis sur les systèmes intégrés d'habitat couvrant les $80 \%$ restants du territoire du pays, divisés en 1600-1700 communes et env. 600 villes restées en dehors des agglomérations.

Les communes particulières ou les groupements de plusieurs communes et villes (surtout petites) peuvent être considérées comme les systèmes locaux d'habitat. Leur caractère local se manifeste par le fait que les unités particulières dhabitat telles que villages, hameaux, écarts et petites villes entretiennent des rapports permanents. Le caractère local résulte de la proximité du lieu d'activités, du voisinage direct ainsi que des liens quotidiens et permanents, et des interdépendances de chaque segment de ce groupement créé par les gens, les entreprises et les institutions publiques.

Dans les territoirs respectifs de 600 communes se trouvent les villes qui fonctionnent comme centres de petites régions. Dans les autres communes, il n'y a pas de villes. Étant donné qu'en Pologne il y a un critère administratif de définition des villes, nous pouvons admettre qu'encore 150 cités au moins se distinguent par un degré élevé d'urbanisation. Ceci nous permet de constater qu'env. 800 cités remplissent la fonction de centres de petites régions formant les systèmes locaux d'habitat. Les fonctions principales de ces régions sont la production alimentaire, celle des matières premières animales et végétales, les fonctions résidentielles et récréatives. Les 800 systèmes locaux que nous venons de citer devraient prochainement faire l'objet des recherches géographiques. Il s'agit surtout des villes centres de ces systèmes et, en premier lieu, des cités urbaines de moins de 20000 habitants. Il y a peu de temps encore, ces centres jouaient un rôle important dans l'organisation de la vie socio-économique - c'étaient les intermédiaires entre la campagne et le marché national, en même temps que les lieux d'approvisionnement en produits et services de toute sorte. Depuis, les progrès s'étant opérés dans la division sociale du travail, une spécialisation dans la production et les services ainsi que le développement des transports ont considérablement modifié leur rôle. En Pologne, ces transformations ont été liées avec la centralisation de nombreux domaines de la vie et avec l'ainsi dite socialisation (nationalisation) du commerce, de l'équipement et de la production. Ces transformations sont à l'origine de l'ainsi dite crise de petites villes dont le résultat est la destruction assez importante des systèmes locaux d'habitat. Jusqu'aujourd'hui, on n'a pas pu surmonter cette crise.

Les problèmes des relations ville - arrière-pays ont eu droit à de nombreuses publications. Les recherches ont fait ressortir l'influence que la ville exerce sur la population (répartition, dynamique de la population, différentes structures démographiques), sur le degré de la satisfaction des besoins de la population et le caractère de ces besoins, sur l'organisation de 
la production et des différentes manifestations de la vie de société ainsi que sur les progrès de l'urbanisation dans les terrains voisins de la ville. Les conditions socio-économiques en train de se former imposent les recherches qui décideraient si les relations et les liens attestés dans le passé sont toujours actuels ou, au contraire, leur caractère a changé et dans quelle mesure.

Les recherches effectuées par M.Jerczyński et K.Dziewoński ont démontré que certaines petites villes étaient choisies par des migrants ruraux comme lieux de séjour temporaire. En effet, l'échange des populations dans ces cités était plus grand qu'il ne résultait des statistiques. Les recherches effectuées dans la région de Suwałki ont fait ressortir d'importants changements de la structure biologique, surtout dans certaines tranches d'âge. Ainsi, la campagne se masculinisait tandis que les villes centres locaux où les services et l'administration publique étaient les activités dominantes - se féminisaient. Par contre, la situation dans le Bassin d'Extraction du Cuivre de Lubin était diamétralement opposée c'était la campagne qui était féminisée tandis que les villes se caractérisaient par une supériorité numérique d'hommes.

Les villes exerçaient toujours une influence essentielle sur la répartition des populations et la stabilité de la colonisation. Les recherches menées par J.Grucza dans la partie Est de la Poméranie Occidentale le démontrent clairement. Par contre, la pénurie de services qui étaient principalement localisés dans les villes est à l'origine de mouvements migratoires assez importants que l'on observe dans les territoires ruraux et, ce qui s'ensuit, du dépeuplement de la campagne (les cas des voïvodies de Koszalin et de Słupsk). On peut dire alors qu'une cité abondonnée, dépeuplée, sort du système, que là-bas, le système s'est dégradé.

Les systèmes locaux d'habitat situés dans l'enceinte des agglomérations urbaines ou dans le voisinage immédiat de grandes villes se forment, se développent et fonctionnent différemment que ceux présentés plus haut. Là, le développement d'un centre régional dépend moins de la nécessité de satisfaire les besoins d'une petite région que de l'impact d'un stimulant venant soit du centre de l'agglomération soit de la grande ville. Tel est le cas, p.ex., de Konstancin-Jeziorna, de Góra Kalwaria ou encore de Karczew, cités dont l'histoire est assez longue, qui se développaient sous l'influence de Varsovie. Également les cités rurales situées autour de ces villes, sont soumises à l'influence directe de la métropole. Nous pouvons même affirmer que l'influence de Varsovie a nivelé, dans un certain degré, la différenciation hiérarchique des cités en enlevant à un centre local une grande partie d'attraction. Les transformations à l'intérieur du système d'habitat dans un territoire rural subissent, dans un degré moindre, l'influence des fonctions remplies par le centre local mais s'opèrent plutôt sous celle des facteurs tels que, p.ex. les transports suffisamment développés permettant de joindre Varsovie vite et facilement. Z.Pióro qui analysait les comportements spatiaux des habitants de l'agglomération varsovienne a attiré l'attention 
sur une sorte de vide (dépression) dans le domaine du commerce et des services observé dans la région située à une certaine distance de Varsovie. Ce vide a pu se former car les habitants de la banlieue varsovienne préféraient satisfaire les besoins dans les domaines de l'approvisionnement et des services à Varsovie plutôt que faire les démarches afin de faire installer ce qui leur faut sur place. Ces derniers temps, cette situation change. Dans la banlieue varsovienne, aussi bien dans les villages que dans les villes, de nombreux établissements et institutions d'utilité publique se sont installés qui travaillent pour les besoins de la métropole - ce sont les commerces (le plus souvent en gros), ateliers de réparation et ateliers de production. Les transformations en cours influeraient certainement sur le développement du système. Il est à noter que les transformations les plus importantes s'opèrent, avant tout, en fonction de la situation d'une cité par rapport aux voies de communication, surtout celles qui mènent à Varsovie.

Les systèmes d'habitat dans les terrains touristiques et dans les stations thermales fonctionnent tout différemment. Les villes remplissent d'un côté le rôle de centres de services spécialisés, adressés aux touristes et estivants, et de l'autre - elles propagent les dernières nouveautés du domaine de l'organisation de la récréation.

Les changements que l'on observe en Pologne, et surtout les tendances à libérer les forces cachées jusqu'à ce temps dans les systèmes locaux influeraient sur la création de nouvelles formes et structures d'habitat. Ainsi, p.ex., un développement des services devrait engendrer les conditions favorisant la création de nouveaux emplois ce qui, à son tour, contribuerait à Yamélioration de la qualité de la vie dans les localités concernées et renforcerait la stabilité du peuplement.

A l'époque de ces transformations historiques, les systèmes locaux d'habitat présentant différents niveaux de formation ou de maturité changeront, eux aussi. Ils pourraient fonctionner correctement seulement si des systèmes et structures adéquats, consécutifs au développement, étaient mis en place. Un tel développement pourrait être encouragé par la mise en vigueur de normes juridiques appropriées, par une supervision conséquente de travaux architectoniques et de construction, enfin par une coopération de toutes les entreprises installées dans un terrain donné. Toutes ces initiatives devraient être préparées et précédées par une reconnaissance de la structure des systèmes d'habitat locaux et des mécanismes de leur fonctionnement. 